Original Article

\title{
Awareness amongst Audiologists Regarding Effects of Radiation Therapy on Auditory System
}

\author{
Bennet Elsa Joseph ${ }^{1}$, Nituna Vinod ${ }^{2}$, Dona Maria Thomas ${ }^{2}$, Arya Krishnan S ${ }^{2}$ \\ ${ }^{1}$ Lecturer, ${ }^{2}$ Students, Nitte Institute of Speech and Hearing, Mangaluru. \\ Corresponding Author : Bennet Elsa Joseph, Lecturer, Nitte Institute of Speech and Hearing, Mangaluru. \\ Mobile :+919967750287 E-mail : bennetelsa@gmail.com
}

Received

: 01.11.2018

Review Completed : 15.11.2018

Accepted

21.11.2018

Keywords : Audiologists, radiation therapy, hearing loss, auditory symptoms

\begin{tabular}{|c|}
\hline Access this article online \\
\hline Quick Response Code \\
\hline
\end{tabular}

\begin{abstract}
Background: Radiation therapy has been a life saver for patients suffering from cancer. Hearing loss can be one of the many side effects of radiation therapy. It can affect the integration of the patient into society after the treatment of cancer is complete. Along with hearing loss, the patients may also experience other auditory symptoms like tinnitus, loudness intolerance and speech perception difficulties which are to be assessed, diagnosed and treated by Audiologists. In order to provide effective rehabilitation, it is necessary that Audiologists are aware of the effect of radiation therapy on the auditory system and what role they play in the interdisciplinary approach. The aim of the present study was to briefly assess whether this awareness is present amongst Audiologists.

Method: A questionnaire was prepared to comprise of questions related to the various aspects of effects of radiation therapy on the auditory system. The questionnaire was circulated online amongst Audiologists and completed questionnaires were subjected to descriptive statistical analysis.

Results : Only 76.9\% Audiologists who were part of the survey were sure that hearing loss can be a side effect of radiation therapy. Many of the Audiologists were not sure whether they should provide audiological services to the patient before radiation therapy or after radiation therapy. $44 \%$ were not sure about what measures could be taken to reduce the effect of radiation therapy on the auditory system. $96.3 \%$ stated that there is a need to receive more specific training in dealing with rehabilitative cases post radiation therapy.
\end{abstract}

\section{Introduction}

Globally, head and neck cancer is estimated to be one of the most common types of cancer. 1 It can be lifethreatening and it is necessary to try to eliminate or reduce the fatality. With the advances in assessment protocols, the identification of the site of lesion and stage of cancer has become more effective and faster. This has aided medical professionals to consider the various intervention options available and weigh the benefits and risks involved with each intervention method. The treatment methods for cancer include surgery, chemotherapy and/or radiation therapy. Radiation therapy involves the use of invisible high energy rays or beams of subatomic particles to damage cancer cells and can stop them from growing and dividing.2 These treatment methods may affect the auditory system and may cause temporary or permanent hearing loss.3
Radiation therapy for head and neck cancer affects the structures of the auditory system in a varying manner and to varying extent amongst patients. The effects that are reported in the literature are inflammation of tissue, earwax blockages, fluid buildup and stiffening of the bones in the ear, damage to the cochlear hair cells and the vestibular system. 3,4 This can result in a conductive, sensorineural or mixed hearing loss and mostly the degree of hearing loss is worse at higher frequencies. 5,6 If identified in the initial stages, the hearing loss can be reversible and can be treated effectively.4 Hearing loss can be unilateral or bilateral depending on various factors like the site of lesion, the dosage of radiation therapy, whether it is in combination with chemotherapy and number of sessions of radiation already taken. The other associated symptoms are tinnitus, speech in noise perception, nausea 
or vomiting and ear pain which may vary from individual to individual.7

After the life-saving contribution of medical treatment is over and the patient is cancer-free and ready to integrate back into society, they might become concerned about the hearing loss as it will affect their quality of life. .8 Many patients may not have been counseled regarding the deteriorating effect of radiation therapy on the auditory and vestibular system which makes acceptance of hearing loss more difficult for the patient.9 This may further hinder the prognosis of the auditory rehabilitation process. As mentioned above, the presence of hearing loss in patients undergoing radiation therapy depends on the dosage, the number of sessions required to complete treatment and other treatment-related factors. There may be other predisposing factors like the occupation of the patient, his family history, the presence of other metabolic disorders, intake of ototoxic drugs, etc. This makes the hearing loss multi-factorial and complex to varying degrees. Hence the hearing loss may vary from patient to patient in terms of progression, type, recovery, the effectiveness of rehabilitative measures, the effect on person's quality of life and other aspects.6,10

Audiologists are professionals who are trained to assess, treat and rehabilitate these hearing and balance disorders. In tertiary hospital set-ups, Audiologists come across cases with hearing loss secondary to radiation. As a part of the curriculum, the effects of radiation on the auditory system may not be taught in many institutions. So Audiologists may not be fully aware of how auditory disorders secondary to radiation can adversely affect the patient and what role they play in the assessment and management of these cases. They may also lack practical knowledge about how to handle these sensitive cases. So a need was felt to understand the level of awareness present amongst the Audiologists regarding these aspects.

\section{Method}

A questionnaire was designed to collect the responses of Audiologists regarding the effect of radiation therapy on the auditory system and their role in the rehabilitation of patients who are at risk of hearing loss secondary to the radiation therapy. 9 questions were of multiple choices format and one question was an open-ended question. The questionnaire was checked for face validity by 5 Audiologists. After receiving approval from the Scientific and ethical committee of the Institution, the questionnaire was converted into Google form and forwarded to the Audiologists through social media. Participants were informed that incomplete forms would be excluded from the study.

Data was collected by snowball sampling and descriptive statistics was used to interpret the data that was collected.

\section{Results}

A total of 110 completed questionnaires were received over a period of 1 week. The frequency of occurrence of the responses and percentages were calculated.

77.3\% Audiologists were aware that radiation therapy may lead to hearing loss, $20.9 \%$ were not sure and $1.8 \%$ responded that it does not lead to hearing loss. As seen in Figure 1, 52.7\% Audiologists feel that the progression of hearing loss secondary to radiation therapy can vary from person to person, $25.5 \%$ think it will be progressive in nature over time, $4.5 \%$ feel its non-progressive while $17.3 \%$ stated they were not sure. Figure 2 shows the responses for the question regarding what type of hearing loss may be expected post radiation therapy. Majority of Audiologists $(60.9 \%)$ think that the patients will have sensorineural hearing loss post-radiation therapy. Other than hearing loss, Audiologists reported that tinnitus might be the second most common symptom reported by these patients along with vertigo, ear block, ear pain, difficulty with speech understanding, loudness intolerance and other symptoms (Figure 3).

$56.5 \%$ Audiologists stated that they were not sure whether the auditory and vestibular symptoms experienced by the patients are reversible or not after the termination of radiation, $11.8 \%$ feel its reversible while $31.8 \%$ stated that it is irreversible. The responses of Audiologists related to what measures do they think may help reduce the effects 
of radiation therapy are shown in Figure 4. According to Audiologists, reduction of dosage, regular follow-ups for monitory hearing sensitivity, use of ear protective devices (EPD) in the noisy situation, prescribing amplification devices with adequate gain were some of the measures. However, $44 \%$ reported that they were not sure.

Out of 110 Audiologists, $56.4 \%$ said that Audiologists should be a part of the pre-therapy assessment team, $24.5 \%$ were not sure and $19.1 \%$ responded they are not. $75.5 \%$ reported that Audiologists should be a part of postradiation assessment and rehabilitation team, $19.1 \%$ were not sure and 5.5\% responded no. $96.4 \%$ stated that importance of counseling the patient about the risk of hearing loss secondary to radiation therapy is paramount. 96.4\% Audiologists want to be trained to acquire more information about how to tackle the cases who are at risk of hearing loss secondary to radiation therapy.

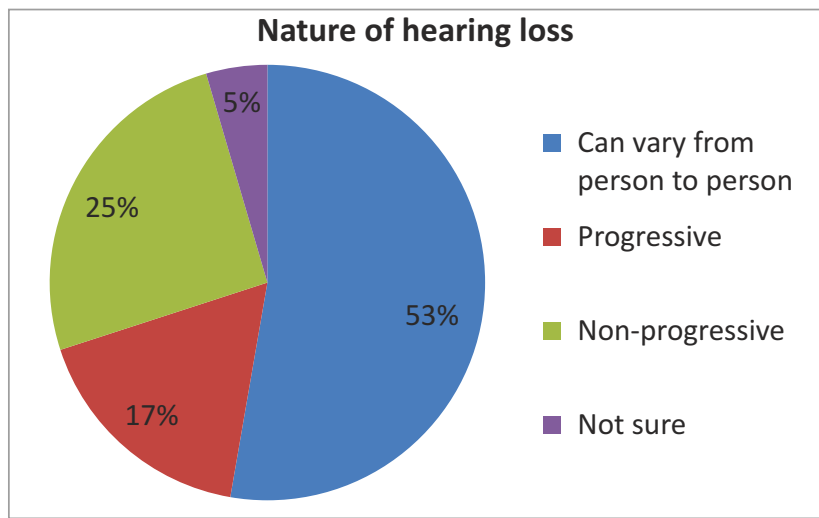

Figure 1 : Responses of Audiologists for nature of hearing loss post radiation therapy

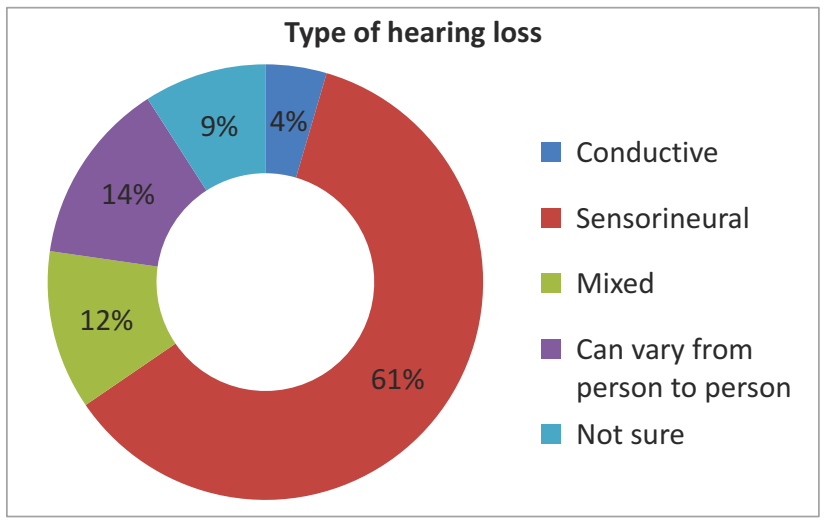

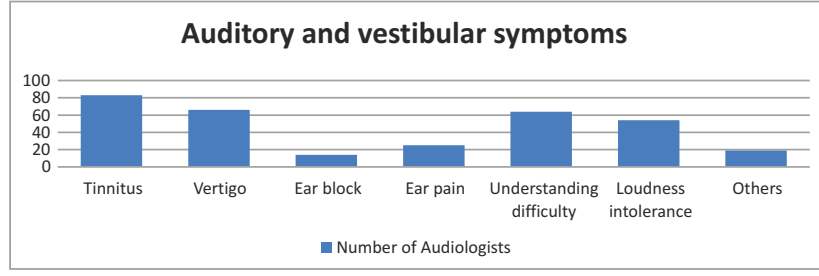

Figure 3 : Responses of Audiologists for auditory and vestibular symptoms other than hearing loss post radiation therapy

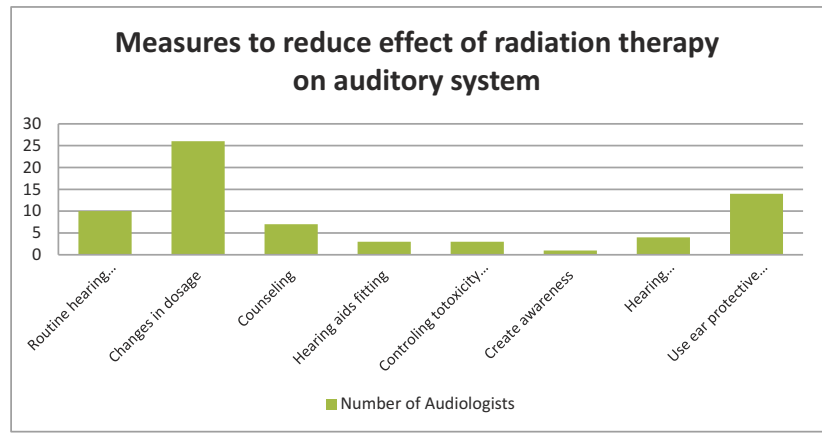

Figure 4 : Measures to reduce effect of radiation therapy on auditory system

\section{Discussion}

Radiation therapy can be considered as one of the major iatrogenic causes of hearing loss in cancer patients especially in those with head and neck cancer. 3 Audiologists should be aware of the various aspects related to hearing loss secondary to radiation therapy. This is of utmost importance when Audiologists have to assess, diagnose and rehabilitate these patients. Many of the studies in literature about the nature of hearing loss state that it is multifaceted and may not be conclusive due to multi-factorial and multi-component nature of hearing loss. So Audiologists have to carefully analyze the factors related to each individual to detect an existing hearing loss or to determine whether they are at risk of acquiring hearing loss as the radiation therapy progresses. These factors will also vary the progression of hearing loss in each individual and from the responses obtained, $52.7 \%$ Audiologists are aware of it.

Most of the studies state that though the different structures and functions of the auditory system may be affected by radiation, the most common type of hearing loss seen at the earliest stage is the sensorineural hearing loss which was correctly answered by $60.2 \%$ Audiologists. $.7,8,10$ Not all participants of this study were aware of the 
various auditory and vestibular symptoms that the patients may experience post radiation therapy. This is important because along with hearing loss, even the other auditory and vestibular symptoms can also affect the quality of life of the patient.4,7,11 Audiologists should be aware of these symptoms as they have to work to resolve these symptoms in coordination with the ENT specialist or other professionals.

$11.8 \%$ Audiologists feel that the effects of radiation therapy that are mentioned above can be reversible. Audiologists need to understand that identification and intervention of hearing loss at the earliest stage possible is necessary as the symptoms may be reversible in the initial stages. 4 During pre-radiation therapy counseling, it is necessary to emphasize to the patient and the family members that routine monitoring of hearing sensitivity is essential as there is a chance to reduce or reverse the effects of radiation therapy.

$44 \%$ of the Audiologists are not sure which measures may actually benefit the patients who have the adverse effect of radiation therapy on their auditory system. The responses of the 110 Audiologists comprises of steps/measures to be taken as the preventive strategy before the treatment begins, measures to be taken during the actual treatment procedure and steps to be taken post radiological intervention. Radiation therapy is a life-saving procedure and at times it may be difficult or almost impossible to alter the dosage or the intervention protocol as suggested by some Audiologists. However, studies do show that reduction in dosage can reduce the initial induction of the side effects.4,12,13 However, empathetic and informative counseling sessions along with routine audiological monitoring may be the best way to keep tabs on the auditory or vestibular changes that the patient is experiencing. As suggested by some of the Audiologists, trying to control the other contributing factors to hearing loss like noise exposure or ototoxicity from other medications may provide a chance to reduce the progression and negative impact on the auditory system. Use of amplification devices is one way to ensure that the deteriorating effects of radiation therapy can be tackled. Radiation therapy can cause a temporary or permanent damage to the outer hair cells and the middle ear reflex mechanism thus creating a higher risk of noise-induced hearing loss for the individual. Use of ear protective devices will help to cut down the risk of noise-induced hearing loss whenever the patient is exposed to noise either at work or in their daily routine.

Very few Audiologists are aware that they have a role to play in the pre-radiation therapy stage of treatment. The baseline audiological evaluation is essential as it will help the Audiologists to identify the predisposing factors and any pre-existing hearing loss. This helps in counseling the patient regarding their present audiological condition and giving them information about what changes in hearing they can expect to experience post radiation therapy and what rehabilitative options may be available for them. The results of the study reveal that not all Audiologists know that they have a role to play in the post radiation therapy assessment and rehabilitation also. This will hinder the entire post-therapy assessment and rehabilitation procedure as the Audiologists will not be well prepared to handle these cases if the patient acquires auditory or vestibular problems. This lack of awareness about their own role in providing audiological clinical services to cancer patients may be because some of them are not working in a hospital setup or because they have not been involved in the interdisciplinary/ multidisciplinary approach of intervention. Lack of adequate training to treat cancer patients who experience auditory or vestibular problems may be another major reason for this lack of awareness.

When diagnosed with cancer, the patient and the family may be emotionally devastated. They will be focused on the intervention to save the life of the person. However, they may not be aware of the other complications or problems which may develop secondary to treatment. So when the side effects start to negatively impact the patient's life, they will be not aware of the assessment and treatment procedures that may help the patient. Hence 
counseling by Audiologists regarding the auditory or vestibular problems at different stages of the treatment will play a major role in making the patient aware of the risks and thereby slowly molding them to overcome these problems in a more effective way.

The results of this study depict that $96.4 \%$ Audiologists are willing to be trained to become more professionally competent to handle these cases. Focused training pertaining to the cases with hearing loss secondary to radiation therapy will lead to more professional outlook amongst Audiologists and help them to render tailored rehabilitation program for these individuals who may display different adverse effects of varying degrees.

\section{Conclusion}

The holistic treatment of cancer is a very sensitive and challenging task for all the professionals involved in the process. The Oncologists play a major role in reducing the high risk of mortality in these patients by means of various management options like radiation therapy. However, an

\section{References}

1. Bray F, Ferlay J, Soerjomataram I, Siegel RL, Torre LA, Jemal A. Global cancer statistics 2018: GLOBOCAN estimates of incidence and mortality worldwide for 36 cancers in 185 countries. CA Cancer J Clin. September 2018. doi:10.3322/caac.21492

2. William C Shiel. Radiation Therapy. https://www.medicinenet. com/radiation_therapy/article.htm\#what_are_the_types_of_radiati on_therapy. Accessed October 24, 2018.

3. Lambert EM, Gunn GB, Gidley PW. Effects of radiation on the temporal bone in patients with head and neck cancer. Eisele DW, ed. Head Neck. 2016;38(9):1428-1435. doi:10.1002/hed.24267

4. Sharad Nilakhe S. Effects of Radiotherapy on Auditory and Vestibular Function Otorhinolaryngology Clinics.; 2014. http://www.jaypee journals.com/eJournals/ShowText.aspx?ID=7173\&Type=FREE\&TYP= TOP\&IID=541\&Value=9\&isPDF=YES. Accessed October 11, 2018.

5. Zhang Y, Lin Z-A, Pan J-J, et al. Concurrent control study of different radiotherapy for primary nasopharyngeal carcinoma: intensitymodulated radiotherapy versus conventional radiotherapy. Ai Zheng. 2009;28(11):1143-1148. http://www.ncbi.nlm.nih.gov/pubmed/ 19895733. Accessed October 24, 2018.

6. Wang L-F, Kuo W-R, Ho K-Y, Lee K-W, Lin C-S. A long-term study on hearing status in patients with nasopharyngeal carcinoma after radiotherapy. Otol Neurotol. 2004;25(2):168-173. http://www.ncbi. nlm.nih.gov/pubmed/15021778. Accessed October 24, 2018.

7. de Oliveira PF, Souza Santos RG, Carneiro TC, et al. Effects in Hearing Thresholds in Subjects Receiving Radiotherapy as Treatment for Head and Neck Cancer. Otolaryngology. 2017;07(02):7-10. doi:10.4172/ interdisciplinary approach is required to bring back the patient's quality of life to a near normal condition.

Hearing loss and other auditory and vestibular problems may restrict the rehabilitation and of the patient back into society and thus affect their quality of life. Audiologists are responsible to provide the services required for these patients. However, as Audiologists lack the adequate training and theoretical knowledge in providing diagnostic and rehabilitative services to the cancer patients, they seem to be unaware of their role, regarding what outcomes or findings they can expect and this will affect effective rehabilitation. Hence, Audiology and Speech-Language Pathology training institutions should focus on providing more experience to their students and professionals by means of additions in curriculum or by organizing seminars/workshops and help update the Audiologists. This will help to improve the quality of services that they can provide to patients and in turn, improve the patients' quality of life.

\section{1-119X.1000299}

8. Schultz C, Goffi-Gomez MVS, Pecora Liberman PH, Pellizzon AC de A, Carvalho AL. Hearing Loss and Complaint in Patients With Head and Neck Cancer Treated With Radiotherapy. Arch Otolaryngol Neck Surg. 2010;136(11):1065. doi:10.1001/archoto.2010.180

9. Kramer SE, Kapteyn TS, Kuik DJ, Deeg DJH. The Association of Hearing Impairment and Chronic Diseases with Psychosocial Health Status in Older Age. J Aging Health. 2002;14(1):122-137. doi:10.1177/ 089826430201400107

10. Hua C, Bass JK, Khan R, Kun LE, Merchant TE. Hearing Loss After Radiotherapy for Pediatric Brain Tumors: Effect of Cochlear Dose. Int J Radiat Oncol. 2008;72(3):892-899. doi:10.1016/j.ijrobp.2008.01.050

11. Gabriele P, Orecchia R, Magnano M, Albera R, Sannazzari GL. Vestibular apparatus disorders after external radiation therapy for head and neck cancers. Radiother Oncol. 1992;25(1):25-30. http://www.ncbi.nlm.nih.gov/pubmed/1410586. Accessed October 11, 2018.

12. Bentzen SM. Preventing or reducing late side effects of radiation therapy: radiobiology meets molecular pathology. Nat Rev Cancer. 2006;6(9):702. doi:10.1038/nrc1950

13. Merchant TE, Conklin HM, Wu S, Lustig RH, Xiong X. Late Effects of Conformal Radiation Therapy for Pediatric Patients With Low-Grade Glioma: Prospective Evaluation of Cognitive, Endocrine, and Hearing Deficits. J Clin Oncol. 2009;27(22):3691. doi:10.1200/JCO.2008. 21.2738 